\title{
Prefronto-cerebellar transcranial direct current stimulation improves visuospatial memory, executive functions, and neurological soft signs in patients with euthymic bipolar disorder
}

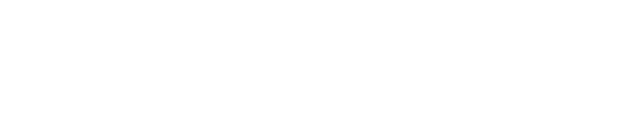

\author{
Amedeo Minichino \\ Francesco Saverio Bersani \\ Laura Bernabei \\ Francesco Spagnoli \\ Lucilla Vergnani \\ Alessandra Corrado \\ Ines Taddei \\ Massimo Biondi \\ Roberto Delle Chiaie \\ Department of Neurology and \\ Psychiatry, Sapienza University \\ of Rome, Rome, Italy
}

\begin{abstract}
Objective: The aim of the study was to improve neuropsychological functioning of euthymic patients with bipolar disorder (BD) using transcranial direct current stimulation (tDCS) applied to cerebellar and prefrontal cortices.

Methods: Twenty-five BD outpatients underwent prefrontal (anodal) and cerebellar (cathodal) tDCS for 3 consecutive weeks. All participants were assessed through the Rey Complex Figure Test delay and copy and the Neurological Examination Scale at baseline and after therapy with tDCS.
\end{abstract}

Results: After tDCS treatment, patients showed significant improvements in visuospatial memory tasks. Patients with worse baseline cognitive performances also showed a significant improvement in executive functioning tasks. Neurological Examination Scale total score and motor coordination subscale significantly improved.

Conclusion: Prefrontal-excitatory and cerebellar-inhibitory stimulations in euthymic BD patients may lead to better neurocognitive performances. This improvement could result from the modulation of prefronto-thalamic-cerebellar circuit activity pattern, which can be disrupted in $\mathrm{BD}$

Keywords: cerebellum, dorsolateral prefrontal cortex, neuropsychology, cognition

\section{Introduction}

Bipolar disorder (BD) is a severe and disabling disease. The course of BD has traditionally been viewed as episodic, ie, with symptomatic, functional, and cognitive recovery between mood episodes. ${ }^{1,2}$ However, clinical evidence recently showed that many BD individuals experience difficulties in daily functioning (eg, higher rates of unemployment and disability than healthy controls) ${ }^{3-5}$ as well as neuropsychological and social deficits even during the euthymic phase of the disease, despite symptomatic mood improvements.

Consistently, an increasing number of clinical observations have been considering the cognitive and social impairments observed in $\mathrm{BD}$ as trait-associated, rather than state-associated, characteristics of the disorder. ${ }^{6-10}$ In particular, visuospatial memory and executive functions have been found to be the neuropsychological domains that are more significantly impaired in euthymic BD patients in comparison with healthy controls. $^{8-10}$

The neurological soft signs (NSS) are minor neurological abnormalities in sensory and motor performances well established in schizophrenia. ${ }^{11,12}$ They have been
Correspondence: Francesco Saverio Bersani

Department of Neurology and Psychiatry, Sapienza University of Rome, Viale dell'Università 30 , Rome, Italy

Tel +393405165865

Fax +390639742364

Email bersani.fs@gmail.com (c) (7) (2) 2015 Minichino et al. This work is published by Dove Medical Press Limited, and licensed under Creative Commons Attribution - Non Commercial (unported, v3.0) License. The full terms of the License are available at http://creativecommons.org/licenses/lby-nc/3.0/. Non-commercial uses of the work are permitted without any further permission from Dove Medical Press Limited, provided the work is properly attributed. Permissions beyond the scope of the License are administered by Dove Medical Press Limited. Information on how to request permission may be found at: http://www.dovepress.com/permissions.php 
described in patients with $\mathrm{BD}^{13,14}$ not only during the mood dysregulation episodes, but also in the euthymic phase of the disease. ${ }^{15}$ In addition, various research has observed deficits in NSS expression and deficits in executive functions, suggesting that the disturbances in the two areas of brain functioning domains may result from the alteration of similar underlying neurobiological substrates. ${ }^{15,16}$

Recent studies hypothesize that cognitive impairments in BD patients could be related to prefronto-thalamiccerebellar circuit dysfunction and, in particular, to the loss of the physiological inverse metabolic activity between the dorsolateral prefrontal cortex (DLPFC) and subcortical areas. ${ }^{17-21}$ In particular, some studies suggest that hypoactivation of the left DLPFC observed in BD could lead to hyperactivation of subcortical structures such as the right cerebellar hemisphere. ${ }^{17,18,20}$ A disruption of prefrontocerebellar circuitry in severe mental disorders was first suggested by Andreasen et al who specifically coined the term of "cognitive dysmetria".22

Transcranial direct current stimulation (tDCS) is a brainmodulating technique using constant, low current delivered directly to the brain areas of interest via inhibitory (cathodal) and excitatory (anodal) electrodes. ${ }^{23-25}$ Intracerebral current flow between the two electrodes excites neurons in the regions of interest, producing both neurophysiological and behavioral changes in the participant. ${ }^{23-25}$ Considering the modulatory properties of $\mathrm{tDCS}$, it is possible to hypothesize that concomitant excitatory stimulation of left DLPFC and inhibitory stimulation of right cerebellar hemisphere might modulate prefronto-cerebellar circuitry, potentially resulting in neurological and neuropsychological improvements.

Previous studies showed that anodal stimulation of DLPFC can improve cognitive performances in both patients and healthy individuals, ${ }^{26}$ while one study showed that cathodal stimulation of cerebellum increased cognitive performances in healthy people. ${ }^{27}$ To the best of our knowledge, anodal stimulation of DLPFC and cathodal stimulation of cerebellum have never been applied: 1) concomitantly and 2 ) in a sample of euthymic bipolar patients.

In the present study we used tDCS on both the right cerebellum hemisphere (cathodal, ie, inhibitory modulation) and left DLPFC (anodal, ie, excitatory modulation) to investigate the effects on visuospatial memory, executive functioning, and NSS performances in euthymic BD patients.

\section{Methods}

\section{Participants and study design}

Twenty-seven outpatients with a diagnosis of BD type I or II referring at the Policlinico Umberto I University Hospital,
Sapienza University of Rome, have been enrolled in the study. Patients were in the euthymic phase of the disorder (this was as assessed by Hamilton Depression Rating Scale score $<7$ and Young Mania Rating Scale <7). ${ }^{28,29}$ Some of these participants had previously been enrolled by our research team in other studies with different aims and objectives. ${ }^{19,30}$

Exclusion criteria were: concomitant neurological diseases, other axis I diagnosis, hospitalization in the last 12 months, left handedness, pharmacological treatment with typical antipsychotics, Hamilton Depression Rating Scale score $>7$, Young Mania Rating Scale $>7$, and mental retardation (IQ <70).

tDCS was administered as an add-on treatment to patients who had been stable on a standard course of pharmacological maintenance therapies for at least 2 months. All participants were assessed at baseline and the day after the last tDCS session.

All participants signed written informed consent. The research protocol was approved by the Ethical Committee of Human Experimentation of Policlinico Umberto I University Hospital and was in accordance with the Helsinki Declaration of 1975.

\section{tDCS}

tDCS was applied through two sponge electrodes (surface area $=25 \mathrm{~cm}^{2}$ ) moistened with a saline solution. The electrode montage was: cathodal tDCS on the right cerebellar cortex, $1 \mathrm{~cm}$ under, and $4 \mathrm{~cm}$ lateral to the inion (approximately comparable to the projection of cerebellar lobule VII onto the scalp); anodal tDCS over the left DLPFC (electrode position was determined by the International 10/20 System for EEG Electrodes, such as that Fp1 corresponded to the DLPFC). The onset and offset of the intervention involved current being increased and decreased, respectively, in a ramp-like manner over 10 seconds. The intensity of stimulation was set at $2 \mathrm{~mA}$ and delivered for 20 minutes every working day (Monday to Friday) for 3 consecutive weeks using a Magstim DC Stimulator Plus and was considered a safe level of exposure, well below the threshold for causing tissue damage. ${ }^{27,31}$

\section{Neuropsychological and neurological assessment}

To test visuospatial memory, participants completed the Rey Complex Figure Test (RCFT) after short and long (immediately and 20 minutes, respectively) delays, while the RCFT copy was used to measure executive functioning. ${ }^{32}$ To minimize the "learning effect", parallel (ie, different) versions of RCFT were used in pre- and post-treatment evaluations.

The neurological examination was performed through the Neurological Evaluation Scale (NES), ${ }^{33}$ which includes 
26 items listing the more frequent and significant NSS. Trained evaluators administered the scales based on the original scoring instructions, and items were scored as 0 (no abnormality), 1 (mild but definite impairment), and 2 (marked impairment). Two items (the suck and snout reflex) were scored 0 (absent) or 2 (present). The results show the total score and the three main subscale scores (sensorial integration, motor coordination, and motor sequencing).

\section{Statistical analysis}

The data were analyzed through SPSS software version 20 .

Data were normally distributed and a paired sample $t$-test was used to compare pre- and post-treatment measures. The significance threshold was $P<0.05$.

\section{Results}

Two patients did not complete the stimulation protocol because of the onset of migraine. The remaining 25 patients tolerated tDCS without complications with the exception of three patients complaining of a transient burning sensation surrounding the electrode site. Demographic and clinical characteristics of these patients are given in Table 1.

Visuospatial memory (measured through the RCFT delay test) improved significantly $(P<0.01)$ after the treatment, although no significant changes were observed in the executive functions domain (measured through the RCFT copy test) (Table 2). NES total score and motor coordination subscale showed significant improvements after tDCS treatment $(P<0.01)$ (Table 2; Figure 1).

To evaluate if the degree of the baseline cognitive impairment in $\mathrm{BD}$ patients could influence the
Table I Clinical and sociodemographic data

\begin{tabular}{|c|c|}
\hline Patients (n) & 25 \\
\hline Age, years (mean \pm standard deviation) & $41.9 \pm 12.62$ \\
\hline \multicolumn{2}{|l|}{ Sex } \\
\hline Males (n) & 8 \\
\hline Females (n) & 17 \\
\hline \multicolumn{2}{|l|}{ Civil status } \\
\hline Married (n) & 8 \\
\hline Single $(n)$ & 11 \\
\hline Others (divorced + widowed) $(n)$ & 6 \\
\hline \multicolumn{2}{|l|}{ Number of years in school } \\
\hline$\leq 9(n)$ & 6 \\
\hline$>9(n)$ & 19 \\
\hline \multicolumn{2}{|l|}{ Diagnosis } \\
\hline Bipolar disorder I (n) & 15 \\
\hline Bipolar disorder II (n) & 10 \\
\hline Disease duration, years (mean \pm standard deviation) & $17.08 \pm 12.05$ \\
\hline Age at onset of illness, years (mean \pm standard deviation) & $28.79 \pm 12.60$ \\
\hline \multicolumn{2}{|l|}{ Psychiatric medication } \\
\hline Lithium (n) & 12 \\
\hline Anticonvulsants (n) & 17 \\
\hline Atypical (n) & 17 \\
\hline Benzodiazepines (n) & 10 \\
\hline Antidepressants (n) & 5 \\
\hline
\end{tabular}

neuropsychological outcomes of the tDCS treatment, we performed supplementary analyses dividing the whole sample into two subgroups of patients: 1) high baseline performances (HBP) group comprised patients with an average baseline score above the median value of the neuropsychological domain of interest; 2) low baseline performances (LBP) group comprised patients with an average baseline score below the median value of the neuropsychological domain of interest. LBP patients showed a more relevant improvement than HBP patients in both visuospatial (RCFT delay test) and executive functions (RCFT copy test) domains (Table 2).

Table 2 Mean scores of neuropsychological and NES assessments pre-tDCS and post-tDCS

\begin{tabular}{|c|c|c|c|}
\hline & Pre-tDCS evaluation & Post-tDCS evaluation & $P$-value \\
\hline \multicolumn{4}{|c|}{ Neuropsychological evaluation } \\
\hline RCFT copy & $26.29 \pm 5.76$ & $28.00 \pm 7.66$ & $0.4 \mathrm{I}$ \\
\hline LBP patients & $21.95 \pm 4.50$ & $27.19 \pm 4.58$ & $0.01 *$ \\
\hline HBP patients & $31.00 \pm 1.99$ & $28.91 \pm 10.41$ & 0.50 \\
\hline RCFT delay & $8.42 \pm 6.22$ & $|3.7| \pm 6.5 \mid$ & $0.01 *$ \\
\hline LBP patients & $3.37 \pm 3.41$ & $9.04 \pm 4.26$ & $<0.0 I^{*}$ \\
\hline HBP patients & $13.37 \pm 4.26$ & $17.74 \pm 5.67$ & 0.06 \\
\hline \multicolumn{4}{|l|}{ NES } \\
\hline Motor coordination & $1.91 \pm 1.54$ & $0.39 \pm 0.65$ & $<0.0 I^{*}$ \\
\hline Motor sequencing & $3.77 \pm 2.84$ & $2.22 \pm 2.6 \mathrm{I}$ & 0.06 \\
\hline Sensory integration & $2.00 \pm 1.48$ & $1.43 \pm 1.85$ & 0.26 \\
\hline Total score & $1 \mathrm{I} .77 \pm 6.48$ & $5.91 \pm 4.44$ & $<0.0 I^{*}$ \\
\hline
\end{tabular}

Note: $* P<0.05$. Data are presented as mean \pm standard deviation.

Abbreviations: HBP, high baseline performances; LBP, low baseline performances; NES, Neurological Evaluation Scale; RCFT, Rey Complex Figure Test; tDCS, transcranial direct current stimulation. 


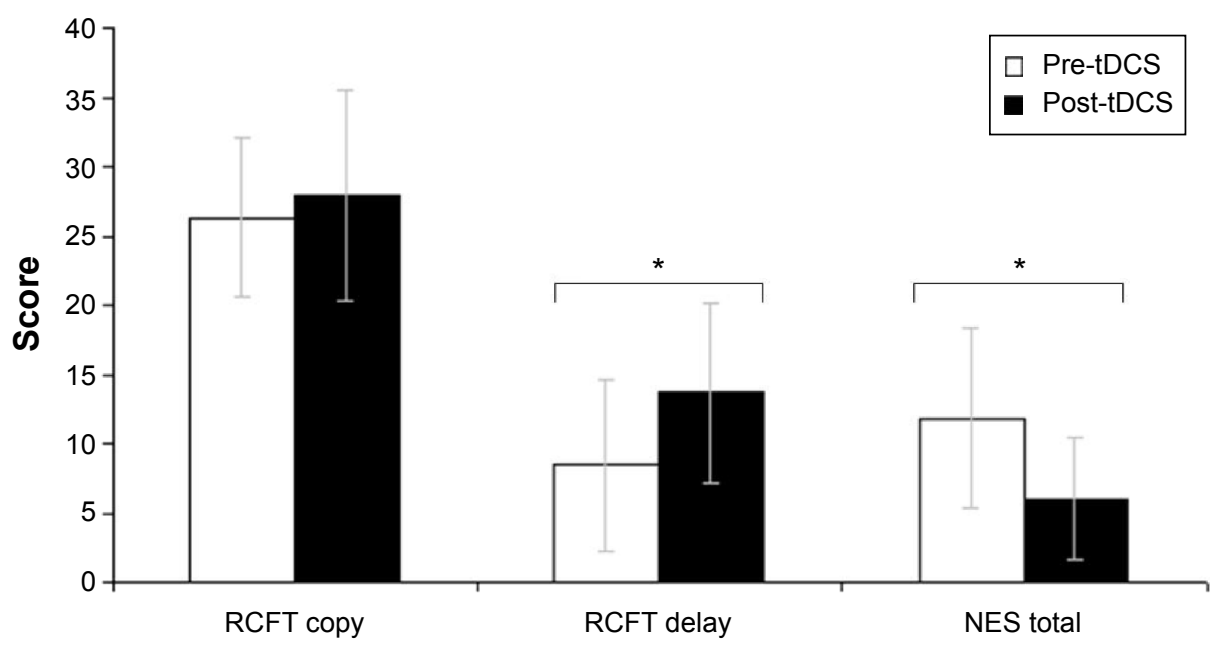

Figure I Mean scores and standard deviations of neuropsychological and NES assessments pre- and post-tDCS.

Note: $* P<0.05$

Abbreviations: NES, Neurological Evaluation Scale; RCFT, Rey Complex Figure Test; tDCS, transcranial direct current stimulation.

\section{Discussion}

To the best of our knowledge, this is the first study investigating the effect of prefronto-cerebellar tDCS on cognitive performances in euthymic $\mathrm{BD}$ patients.

Cognitive deficits have gained considerable importance as critical features of a wide range of psychiatric disorders ${ }^{10,34-38}$ and they post an important therapeutic challenge. ${ }^{7,839}$ While there is overwhelming evidence on the efficacy of psychotropic medications in the management of clinical symptoms, cognitive gains are often poor. ${ }^{7,8,39}$ Thus, it is currently accepted that cognitive impairment and symptomatic manifestations of mental disorders require separate and integrated therapeutic approaches.

The results of the present research preliminarily suggest that concomitant prefrontal-excitatory and cerebellar-inhibitory tDCS might have a positive effect on visuospatial memory and executive functioning in euthymic BD patients. Andreasen's idea of "cognitive dysmetria" hypothesized that a general loss of mental processes coordination could be the consequence of prefronto-thalamic-cerebellar circuit disruption, ${ }^{22}$ and this idea has been supported by several subsequent studies. ${ }^{17,18,20}$ It is thus speculatively possible that the cognitive improvements observed in our patients may be at least partially attributable to a functional modulation of prefronto-cerebellar circuitry activity.

Stratifying the patients according to baseline cognitive functions, we found that LBP patients showed significantly improved executive functions and presented more significant benefits in visuospatial memory than HBP patients. This finding suggests that individuals with more severe cognitive impairments, that are often the most clinically severe and the most difficult to treat, may obtain more substantial benefits from prefronto-cerebellar tDCS.

NSS are indicators of neurological impairment frequently observed in schizophrenia and in other psychiatric disorders, ${ }^{11-15}$ our results showed that prefronto-cerebellar tDCS led to significant improvements of the NES total score and NES motor coordination subscale.

A recent diffusion tensor imaging study found an inverse correlation between NSS severity and the integrity of thalamic-cerebellar tracts ${ }^{40}$ supporting our study hypothesis. Furthermore, Goswami et al recently found a correlation between NSS and executive dysfunctions in euthymic BD patients, potentially involving an inadequate functioning of prefrontal cortex; ${ }^{15}$ this evidence is consistent with our results, as both NSS and executive functions improved after DLPFC stimulation.

\section{Conclusion}

In conclusion, the present study provides preliminary evidence that concomitant prefrontal-excitatory and cerebellarinhibitory tDCS in euthymic BD patients may lead to better neurocognitive performances, quantified through neuropsychological and neurological measures. We suggest that this improvement is at least partially attributable to functional modulations of prefronto-cerebellar circuitry activity, which has been proven to be altered in BD as well as in other psychiatric disorders. Prefronto-cerebellar tDCS could potentially represent an inexpensive, easy to administer, non-invasive, and painless therapy to increase the effectiveness of standard BD treatment. 
The small sample size and the absence of a sham control group are major limitations of the study. In addition, we assessed a relatively large number of measures and we did not correct for multiple comparisons; therefore, these results are exploratory/hypothesis-generating and further studies are needed.

\section{Acknowledgment}

The research was fully funded by the Department of Neurology and Psychiatry of Sapienza University of Rome.

\section{Disclosure}

The authors report no conflicts of interest in this work.

\section{References}

1. Trede K, Salvatore P, Baethge C, Gerhard A, Maggini C, Baldessarini RJ. Manic-depressive illness: evolution in Kraepelin's Textbook, 1883-1926. Harv Rev Psychiatry. 2005;13(3):155-178.

2. Rovai L, Maremmani AG, Rugani F, et al. Do Akiskal and Mallya's affective temperaments belong to the domain of pathology or to that of normality? Eur Rev Med Pharmacol Sci. 2013;17(15):2065-2079.

3. Kessler RC, Akiskal HS, Ames M, et al. Prevalence and effects of mood disorders on work performance in a nationally representative sample of U.S. workers. Am J Psychiatry. 2006;163(9):1561-1568.

4. Kogan JN, Otto MW, Bauer MS, et al. Demographic and diagnostic characteristics of the first 1,000 patients enrolled in the Systematic Treatment Enhancement Program for Bipolar Disorder (STEP-BD). Bipolar Disord. 2004;6(6):460-469.

5. Tohen M, Zarate CA Jr, Hennen J, et al. The McLean-Harvard FirstEpisode Mania Study: prediction of recovery and first recurrence. $A m$ J Psychiatry. 2003;160(12):2099-2107.

6. Bersani G, Polli E, Valeriani G, et al. Facial expression in patients with bipolar disorder and schizophrenia in response to emotional stimuli: a partially shared cognitive and social deficit of the two disorders. Neuropsychiatr Dis Treat. 2013;9:1137-1144.

7. Campanella S. Why it is time to develop the use of cognitive eventrelated potentials in the treatment of psychiatric diseases. Neuropsychiatr Dis Treat. 2013;9:1835-1845.

8. Deckersbach T, Nierenberg AA, Kessler R, et al. RESEARCH: Cognitive rehabilitation for bipolar disorder: An open trial for employed patients with residual depressive symptoms. CNS Neurosci Ther. 2010;16(5): 298-307.

9. Martínez-Arán A, Vieta E, Colom F, et al. Cognitive impairment in euthymic bipolar patients: implications for clinical and functional outcome. Bipolar Disord. 2004;6(3):224-232.

10. Robinson LJ, Thompson JM, Gallagher P, et al. A meta-analysis of cognitive deficits in euthymic patients with bipolar disorder. $J$ Affect Disord. 2006;93(1-3):105-115.

11. Dazzan P, Murray RM. Neurological soft signs in first-episode psychosis: a systematic review. Br J Psychiatry Suppl. 2002;43: s50-s57.

12. Bersani G, Clemente R, Gherardelli S, Bersani FS, Manuali G. Obstetric complications and neurological soft signs in male patients with schizophrenia. Acta Neuropsychiatr. 2012;24(6):344-348.

13. Nasrallah HA, Tippin J, McCalley-Whitters M. Neurological soft signs in manic patients. A comparison with Schizophrenic and control groups. $J$ Affect Disord. 1983;5(1):45-50.

14. Goswami U, Basu S, Khastgir U, et al. Neurobiological characterization of bipolar affective disorders: a focus on tardive dyskinesia and soft neurological signs in relation to serum dopamine Beta hydroxylase activity. Indian J Psychiatry. 1998;40(3):201-211.
15. Goswami U, Sharma A, Khastigir U, Ferrier IN, Young AH, Gallagher P, et al. Neuropsychological dysfunction, soft neurological signs and social disability in euthymic patients with bipolar disorder. Br J Psychiatry. 2006;188:366-373.

16. Dimitri-Valente G, Rigucci S, Manfredi G, Girardi P, Ferracuti S. Neurological soft signs: meaning and relevance along the course of psychiatric illness. An objective and rapid screening for psychosis? Riv Psichiatr. 2012;47(6):465-478. Italian.

17. Benson BE, Willis MW, Ketter TA, et al. Interregional cerebral metabolic associativity during a continuous performance task (Part II): differential alterations in bipolar and unipolar disorders. Psychiatry Res. 2008;164(1):30-47.

18. Kimbrell TA, Ketter TA, George MS, et al. Regional cerebral glucose utilization in patients with a range of severities of unipolar depression. Biol Psychiatry. 2002;51(3):237-252.

19. Minichino A, Bersani FS, Spagnoli F, et al. Prefronto-cerebellar transcranial direct current stimulation improves sleep quality in euthymic bipolar patients: a brief report. Behav Neurol. 2014;2014:876521.

20. Minichino A, Bersani FS, Trabucchi G, et al. The role of cerebellum in unipolar and bipolar depression: a review of the main neurobiological findings. Riv Psichiatr. 2014;49(3):124-131.

21. Delle Chiaie R et al., Bipolar spectrum disorders in patients with cerebellar lesions. a comparison with Parkinson's disease. Journal of Nervous and Mental Disease 2015. In press.

22. Andreasen NC, Nopoulos P, O'Leary DS, Miller DD, Wassink T, Flaum M. Defining the phenotype of schizophrenia: cognitive dysmetria and its neural mechanisms. Biol Psychiatry. 1999;46(7):908-920.

23. Bersani FS, Biondi M. Historical recurrences in psychiatry: somatic therapies. Riv Psichiatr. 2012;47(1):1-4. Italian.

24. Medeiros LF, de Souza IC, Vidor LP, et al. Neurobiological effects of transcranial direct current stimulation: a review. Front Psychiatry. 2012;3:110

25. Poreisz C, Boros K, Antal A, Paulus W. Safety aspects of transcranial direct current stimulation concerning healthy subjects and patients. Brain Res Bull. 2007;72(4-6):208-214.

26. Mondino M, Bennabi D, Poulet E, Galvao F, Brunelin J, Haffen E. Can transcranial direct current stimulation (tDCS) alleviate symptoms and improve cognition in psychiatric disorders? World J Biol Psychiatry. 2014;15(4):261-275.

27. Pope PA, Miall RC. Task-specific facilitation of cognition by cathodal transcranial direct current stimulation of the cerebellum. Brain Stimul. 2012;5(2):84-94.

28. Hamilton M. A rating scale for depression. J Neurol Neurosurg Psychiatry. 1960;23:56-62.

29. Young RC, Biggs JT, Ziegler VE, Meyer DA. A rating scale for mania: reliability, validity and sensitivity. Br J Psychiatry. 1978;133: 429-435.

30. Bersani FS, Minichino A, Fattapposta F, Mannarelli D, Pauletti C, Imperatori C, et al. P300 component in euthymic patients with bipolar disorder type I, bipolar disorder type II and healthy controls: a preliminary event-related potential study. Neuroreport. 2015;26(4): 206-210.

31. Boggio PS, Ferrucci R, Rigonatti SP, et al. Effects of transcranial direct current stimulation on working memory in patients with Parkinson's disease. J Neurol Sci. 2006;249(1):31-38.

32. Shin MS, Park SY, Park SR, Seol SH, Kwon JS. Clinical and empirical applications of the Rey-Osterrieth Complex Figure Test. Nat Protoc. 2006;1(2):892-899.

33. Buchanan RW, Heinrichs DW. The Neurological Evaluation Scale (NES): a structured instrument for the assessment of neurological signs in schizophrenia. Psychiatry Res. 1989;27(3):335-350.

34. Castellanos FX, Sonuga-Barke EJ, Milham MP, Tannock R. Characterizing cognition in ADHD: beyond executive dysfunction. Trends Cogn Sci. 2006;10(3):117-123.

35. Heinrichs RW, Zakzanis KK. Neurocognitive deficit in schizophrenia: a quantitative review of the evidence. Neuropsychology. 1998;12(3): 426-445. 
36. Loeber S, Vollstädt-Klein S, von der Goltz C, Flor H, Mann K, Kiefer F. Attentional bias in alcohol-dependent patients: the role of chronicity and executive functioning. Addict Biol. 2009;14(2):194-203.

37. Mossner R, Mikova O, Koutsilieri E, et al. Consensus paper of the WFSBP Task Force on Biological Markers: biological markers in depression. World J Biol Psychiatry. 2007;8(3):141-174.

38. Bersani FS, Minichino A, Fojanesi M, Gallo M, Maglio G, Valeriani G, et al. Cingulate Cortex in Schizophrenia: its relation with negative symptoms and psychotic onset. A review study. Eur Rev Med Pharmacol Sci. 2014;18(22):3354-3367.
39. Larøi F, Raballo A. Notes from Underground: Are Cognitive-Enhancing Drugs Respecting their Promises? Front Psychol. 2010;1:158.

40. Mittal VA, Dean DJ, Bernard JA, et al. Neurological soft signs predict abnormal cerebellar-thalamic tract development and negative symptoms in adolescents at high risk for psychosis: a longitudinal perspective. Schizophr Bull. 2014;40(6):1204-1215.

\section{Publish your work in this journal}

Neuropsychiatric Disease and Treatment is an international, peerreviewed journal of clinical therapeutics and pharmacology focusing on concise rapid reporting of clinical or pre-clinical studies on a range of neuropsychiatric and neurological disorders. This journal is indexed on PubMed Central, the 'PsycINFO' database and CAS, and is the official journal of The International Neuropsychiatric Association (INA). The manuscript management system is completely online and includes a very quick and fair peer-review system, which is all easy to use. Visit http://www.dovepress.com/testimonials.php to read real quotes from published authors.

Submit your manuscript here: http://www.dovepress.com/neuropsychiatric-disease-and-treatment-journal 